\title{
Modeling the effect of produced water on asphaltene deposition in a production tubing using population balance
}

\author{
R. Mollaabbasi \\ Chemical engineering department \\ Université Laval \\ Québec, Canada \\ Roozbeh.mollaabbasi.1@ulaval.ca
}

\author{
D. Eskin \\ Schlumberger-Doll Research \\ Cambridge, USA
}

\author{
S. M. Taghavi \\ Chemical engineering department \\ Université Laval \\ Québec, Canada
}

\begin{abstract}
An engineering model evaluates the effect of produced water in a production tubing on the reduction of asphaltene deposition on the tube (pipe) wall. We rely on an industrial hypothesis that droplets colliding with the pipe partially prevent the deposition of the asphaltene particles on the wall surface. First, we use a population balance model to calculate the droplet size evolution along a production tubing. Then, we study the fraction of the pipe wall surface dynamically coated by water droplets. To do so, we consider the wall bombardment by droplets that fluctuate in a turbulent flow. The results demonstrate that the dynamically coated area by droplets increases gradually ( $\max$ by order of $10^{-4}$ ), the effects of which on asphaltene deposition is negligible.
\end{abstract}

Keywords-Asphaltene $\quad$ precipitation; droplet breakup;
population balance model

\section{INTRODUCTION}

The interaction between asphaltene particles and droplets is an important problem of flow assurance in the oil industry. Asphaltenes are molecular substances, which compose crude oil along with resins, aromatic hydrocarbons and saturated hydrocarbons such as alkanes that are not soluble in straightchain solvents. Asphaltenes precipitate from a hydrocarbon fluid when the local pressure drops below the so-called asphaltene onset precipitation pressure. Further downstream pressure decrease is accompanied with further precipitation. Complex deposition phenomenon consists of different processes. Precipitated particles interact with each other, grow due to agglomeration, are transported to the wall and stick to its surface with some probability [1, 2]. Asphaltene deposition represents a significant concern only for a vertical production tubing. Here, a substantial pressure drop required for intense asphaltene precipitation is obtained due to hydrostatic pressure difference. Asphaltene deposition has received significant attention in the literature [3, 4]. Numerous articles have considered the problem of asphaltene deposition in reservoirs $[5,6]$, in pipelines $[1,2]$ and in the Couette flows [1]. Some of published asphaltene deposition models demonstrated a reasonable agreement with experimental data. It is important to emphasize that those models were developed for a single-phase (hydrocarbon) flow, whereas, in practice, water is usually produced along with oil. The effects of droplets on the asphaltene deposition in turbulent flow are an unanswered question due to the physical complexity of the problem.

\section{MODELING}

\section{A. Basic concept of accounting for an effect of dispersed water on asphaltene deposition}

Figure 1 illustrates asphaltene deposition on the tubing wall in the presence of dispersed water. The smaller particles and the larger spheres denote the asphaltene aggregates and the water droplets, respectively. According to the comprehensive approach to asphaltene deposition modeling, this phenomenon is a complex process, which consists of the following components: 1) precipitation of primary asphaltene particles; 2) particle agglomeration; 3) particle transport to the pipe wall; 4) interaction of particles with the wall. Eskin et al. [1,2] showed that the asphaltene deposition rate is a strong function of the particle size. Therefore, modeling of the particle size distribution evolution along a production tubing is a key deposition model element. Brownian motion as well as turbulent diffusion (dispersion) govern both agglomeration and deposition of asphaltene particles. However, Eskin et al. [2] showed that turbulent diffusion can be neglected because only very small submicron particles are able to deposit on the pipe wall.

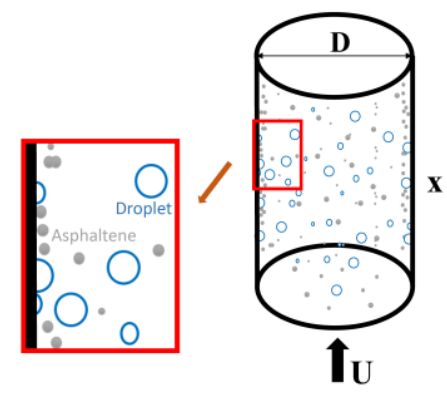

Figure 1. Diagram illustrating asphaltene deposition in a pipe in presence of asphaltene particles and water droplets. 
Here, we consider the effect of water on asphaltene deposition. Water droplets absorb asphaltene molecules. It leads to a modification of droplet surfaces causing coalescence suppression, i.e., droplet stabilization. Non-coalescing droplets are intensely fragmented by turbulence and can reach rather small sizes. At high water holdups ( $50 \%)$, the droplet specific surface may reach very high values. For example, this surface can be up to 3 orders of magnitude larger than the corresponding specific surface of the pipe wall. However, a simple analysis shows the amount of asphaltene absorbed by droplets is small. Indeed, absorbed asphaltene molecules form a monolayer on a droplet surface. The thickness of this monolayer is approximately equal to the diameter of an asphaltene molecule, which does not exceed a few nanometers [3]. Because the monolayer thickness is so small, the total volume concentration of asphaltenes absorbed on droplets is always much lower $\left(<\sim 10^{-4}\right)$ than the volume concentration of asphaltenes in a fluid, which can cause a significant deposition rate $\left(\sim 10^{-2}\right)$ [3]. Therefore, an effect of water on asphaltene deposition is limited to only droplets-pipe wall interactions. Droplets, driven by turbulence eddies, collide with the wall (deposit surface) and bounce from it. In the course of a dropletwall interaction, a deformed droplet coats some surface area of the wall. This area can vary during the bouncing process. Evidently, the area coated by a droplet is unavailable for depositing aspahltene particles. Thus, the problem of accounting for an effect of water on asphaltene deposition can be reduced to determining the mean fraction of the pipe wall surface that is dynamically coated with water droplets. Then, the deposition flux in the presence of dispersed water is calculated as: $q_{a v}=q_{a}(1-\mathrm{S})$, where, $q_{a}$ is the asphaltene deposition flux and $S$ is the mean fraction of the pipe wall surface dynamically coated by water droplets. To determine the parameter $S$ we need to evaluate the droplet size distribution in a pipe flow and consider a process of droplet interaction with the pipe wall (deposit surface).

\section{B. Droplet dispersion in a pipe flow}

We have relied on the population balance model (PBM) in order to predict the droplet size evolution along the time. A disbalance between the viscous stress of the fluid inside the droplets, the surface tension of the droplets and the external stress causes droplet breakup from the continuous phase. The competition of these stresses destroys the surface of the droplets and droplet breakup occurs. A transient diameter based on PBM holding the breakage and the coalescence terms can be written as follows in turbulent uniform conditions $[7,8]$ :

$$
\frac{d f_{i}}{d t}=-B_{D}(\mathrm{i}, \mathrm{t})+B_{B}(\mathrm{i}, \mathrm{t})-C_{D}(\mathrm{i}, \mathrm{t})+C_{B}(\mathrm{i}, \mathrm{t}),
$$

where $f_{i}$ is the mass based distribution of the $i_{t h}$ droplet. $B_{D}, B_{B}, C_{D}$ and $C_{B}$ are the breakage death, the breakage birth, the coalescence death and the coalescence birth of droplets respectively in the PBM model. The breakage death has been defined as:

$$
B_{D}(i, t)=b(i) f(i, t) \text {. }
$$

The breakage frequency function $b(i)$ and daughter size distribution function are investigated by different methods (e.g., Coulaloglou and Tavlarides [9]). The breakage birth is calculated as:

$$
B_{B}(i, t)=v(i) \int_{d_{i}}^{d_{i \max }} h(i, j) b(j) \frac{f(j, t)}{v(j)} d\left(d_{j}\right)
$$

where $v(i)$ is the volume of the $i_{t h}$ droplet and daughter size redistribution function $h(i, j)$ is calculated by Coulaloglou and Tavlarides [9]. The coalescence birth term is:

$$
\begin{aligned}
& C_{B}(i, t)=\frac{d^{2}(\mathrm{i}) v(i)}{2} \times \\
& \int_{d_{\min }}^{\left(d_{i}^{3}-d_{\min }{ }^{3}\right)^{1 / 3}} \frac{C\left(\left[d_{i}^{3}-d_{j}^{3}\right]^{1 / 3}, d_{j}\right)}{\left[d_{i}^{3}-d_{j}^{3}\right]^{2 / 3}} \frac{f(i, t)}{\rho_{d} v(j)} \frac{f\left(\left[d_{i}^{3}-d_{j}^{3}\right]^{1 / 3}, t\right)}{v(i)-v(j)} d\left(d_{j}\right) .
\end{aligned}
$$

The coalescence death term is [10]:

$$
C_{D}(i, t)=f(i, t) \int_{d_{\min }}^{\left(d_{\max } \int^{3} d_{i}^{3 / 3}\right.} C\left(d_{i}, d_{j}\right) \frac{f(j, t)}{\rho_{d} v(j)} d\left(d_{j}\right) .
$$

Figure 2 shows the evolution of pure droplet with time in oil, $\rho_{d}=965.3 \mathrm{~kg} / \mathrm{m}^{3}, \mu_{d}=0.3 \mathrm{mPa} / \mathrm{s}$ and liquid-liquid surface tension is $0.0056 \mathrm{~N} / \mathrm{m}$. We have considered the monodispersed initial condition to solve PBM. The initial mass fraction of droplets is $\phi=0.1$ (assuming a very diluted system to neglect the coalescence terms) and the initial droplet size is $d_{d}=150 \mu \mathrm{m}\left(V_{i+1} / V_{i}=0.5\right.$ and $\left.\mathrm{i}=1: 20\right)$. The results show that the droplet size is alleviated with time. Figure $2 \mathrm{~d}$ illustrates the evolution of the mean droplet size with time in a log-log scale, showing that there are three different regions in the droplet breakage with time in case of initially mono-dispersed droplets.
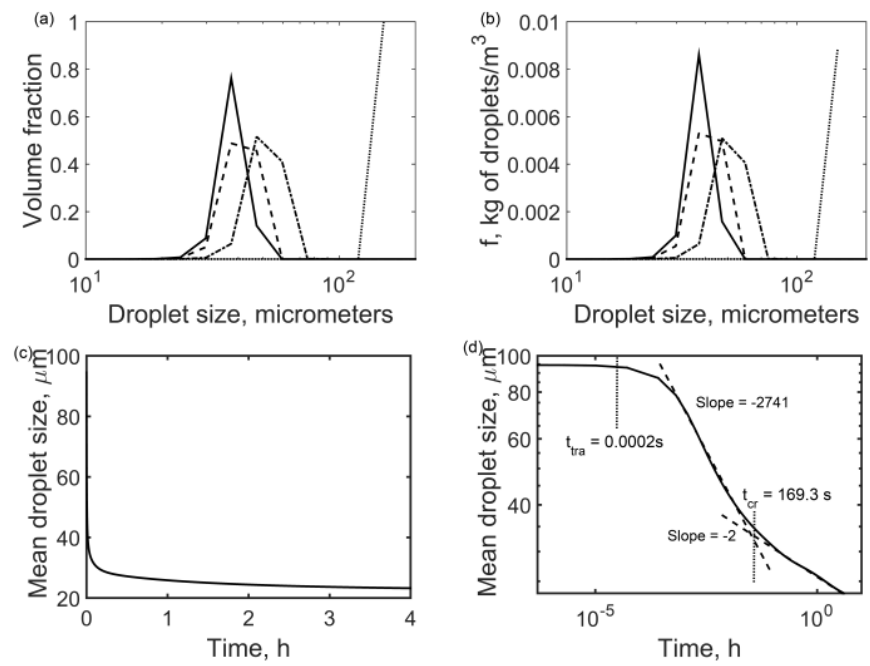

Figure 2. Size evolution of pure droplet with time in turbulent system. Subfigures in the top row show the volume fraction and mass distribution function with time from left to right (the breakage time is $0 \mathrm{~h}, 0.1 \mathrm{~h}, 1.5 \mathrm{~h}$ and $4.0 \mathrm{~h}$ in the dotted line, dash-dotted line, dashed line and solid line respectively). The bottom row shows the average droplet size with time in linear and logarithmic axis. The continuous and dispersed phases are considered as oil and water respectively. 
The mean droplet size does vary much with time in the first region before reaching the transition time $\left(t_{\text {tra }}\right)$, which is in the order of $10^{-4} \mathrm{~s}$. The transition time represents the time required for the imbalance between the viscous stress of the fluid inside the droplet and surface tension to break the droplets considering the mono dispersed droplet distribution as an initial condition. Another important time is the critical time $\left(t_{c r}\right)$ which is quantified by the inflection point of the mean droplet size and it demonstrates the time when the droplet breakup mechanism is changed. Rapid size reduction implies that eddies have more effects on the turbulent mechanism of droplet breakup due to the large size of droplets (first zone). Brownian motion has more effects on collision of droplets in the case of the small droplet size; therefore, the rate of size reduction decreases in tiny droplets. The coalescence terms do not have a significant effect on the PBM in diluted systems because the coalescence terms are 5 orders of magnitude smaller than the breakup terms. Solsvik et al. [8] have stated the coalescence terms are negligible compared to droplet breakup in a dilute dispersed system. The results of our simulation confirm this statement.

\section{Droplet deposition on the wall}

To evaluate the cumulative wall coating effect caused by multiple droplets-wall interactions, a collision of a single droplet with the wall should be considered. In practice, a pipe wall is often characterized by a rather significant roughness. This circumstance complicates the interaction analysis. However, a reasonable estimate of the interaction phenomenon could be obtained assuming that the wall is smooth. The larger the ratio between the droplet diameter and the wall roughness, the closer this is to reality. The only significant mechanism causing a droplet-wall collision is a droplet fluctuation caused by a turbulent eddy. Following Kuboi [11], we assume that droplet fluctuations are mainly controlled by eddies, whose scales are equal to droplet sizes. Usually, the droplet fluctuation is relatively low. Therefore, we assume that the droplet deformation during the interaction process is small. Obviously, after a collision with the wall the droplet should ricochet. Thus, the droplet-wall interaction process consists of the two stages: droplet compression (deformation) and relaxation (shape restoration). Due to the smallness of droplet deformation, we do not consider a hydrodynamic flow field inside the droplet at the compression stage. Then, we can formulate the momentum equation for the droplet-wall interaction, assuming that all fluid elements inside a droplet have the same velocity (see Figure 3). Also, we make additional assumptions to derive the momentum equation for a droplet interacting with the wall. We assume that the droplet interacting with the wall maintains a shape of a sphere with a cut segment. The volume of this segment is neglected due to the assumed deformation smallness. We also assume that during the compression stage, the wall reaction force acting on a droplet is comprised of the two components. First, a hydrodynamic reaction force appears because a droplet interacting with the wall loses momentum component normal to the wall. We evaluate this force by considering an interaction of an imaginary jet of radius a. We consider that this this force is absent during the relaxation stage. The second component of the reaction force is the capillary force applied also to the same droplet wall contact area. For the sake of simplicity, we neglect the drag force acting on the droplet during its interaction with the wall. Because a turbulent eddy transporting the droplet is also decelerated in the wall vicinity, this assumption should not significantly affect the droplet-wall interaction process. Thus, the momentum conservation equation for a droplet of the i-th size fraction interacting with the wall during the compression stage is:

$$
\left(\rho_{d}+\frac{1}{2} \rho_{o}\right) \nu_{i} \frac{d V_{i y}}{d t}=-\rho_{d} V_{i y} \pi a_{i}^{2}-\frac{2 \sigma}{R_{i}} \pi a_{i}^{2} .
$$

$V_{i y}$ is the velocity component normal to the wall for the i-th size fraction droplet. In this equation, the left-hand side represents the inertial force that is equilibrated by the forces expressed by the terms on the right-hand side, the hydrodynamics reaction force and the capillary force, respectively. Let us formulate equation 5 in the dimensionless form as follows:

$$
\frac{d^{2} \tilde{y}_{i}}{d \tau_{i}^{2}}=-\frac{3}{4} \frac{1}{1+s}\left(1-\tilde{y}_{i}^{2}\right)\left(\frac{d \tilde{y}_{i}^{2}}{d \tau_{i}}+1\right),
$$

where $\tilde{y}_{i}=y_{i} / R_{i} \quad, \quad \tilde{V}_{i}=V_{i} / \Omega_{i}, \quad \Omega_{i}=\sqrt{2 \sigma / \rho_{d} R_{i}} \quad$ and $s=\rho_{o} / 2 \rho_{d}$. This equation can be solved numerically considering $\tau_{i}=0, \tilde{y}_{i}=1$ and $\frac{d \tilde{y}_{i}}{d \tau}=\tilde{V}_{i y o}$. We assume that the fluid fluctuation velocity distribution is Maxwellian. In addition, we assume that a droplet exactly follows the fluid in a fluctuation motion. The root-mean-square fluid fluctuation velocity can be calculated by the following empirical correlation [1]:

$$
\sqrt{\bar{V}^{2+}}=\frac{0.005 y^{+2}}{1+0.002923 y^{+2.128}} .
$$

Here, $\bar{V}_{y}^{\prime 2}\left(\frac{d_{i}}{2}\right)$ is the mean-square fluid fluctuation velocity at the moment of touching the wall. $y^{+}=y / \delta^{+}$is the normalized distance to the wall.

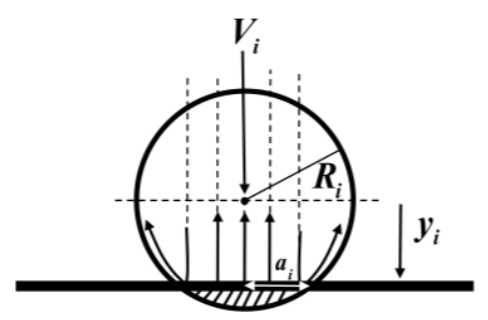

Figure 3. The schematic of the droplet interacting with the wall. 
$\delta^{+}$is the wall layer thickness. Then, it is considered that the initial value of normal to the wall droplet fluctuation velocity is equal to the most probable fluid fluctuation velocity at $y=0.5 d_{i}$. Equation 6 is easily integrated up to the moment when $\tilde{V}_{i y}=0$ indicating the end of the compression stage. The specific pipe wall area dynamically covered by droplets of the i-th size fraction due to their multiple collisions with the wall is: $S_{i}=\operatorname{fr}_{i} S_{i} A_{i}$, where $A_{i}$ is the mean droplet-wall contact area during a single collision; $\zeta_{i}$ is the mean droplet wall contact time; $f r$ is the frequency of droplet-wall collisions. The frequency of droplet-wall collisions per surface area unit is determined, assuming the Maxwellian droplet fluctuation velocity distribution. The total specific area (per unit pipe surface area) covered by droplets is calculated as:

$$
S=\sum_{i=1}^{m} f r_{i}[\underbrace{\int_{R_{i}}^{y_{i f}} \pi\left(R_{i}^{2}-y_{i}^{2}\right) \frac{d y_{i}}{V_{i y}}}_{\text {compression }}+\underbrace{\int_{y_{i f}}^{R_{i}} \pi\left(R_{i}^{2}-y_{i}^{2}\right) \frac{d y_{i}}{V_{i y}}}_{\text {relaxation }}] \text { (9) }
$$

The problem of accounting for the effect of water on asphaltene deposition can be reduced to determining the mean fraction of the pipe wall surface that is dynamically coated with water droplets. Then, the deposit flux in the presence of dispersed water is calculated as: $q=q_{a}(1-\mathrm{S})$ which $q_{a}$ is the asphaltene deposition flux and $S$ is the mean fraction of the pipe wall surface dynamically coated by water droplets.

Let us illustrate the results of the model developed by a few examples. We consider a dispersion of water in oil flowing in a production tubing of the diameter $D=0.0625 \mathrm{~m}$ with the mean velocity $U$. The water holdup is $\phi=0.1$. The oil viscosity and density are $\mu_{o}=1.3 \mathrm{mPa} / \mathrm{s}$ and $\rho_{o}=760 \mathrm{~kg} / \mathrm{m}^{3}$, respectively. The interfacial tension is $0.0025 \mathrm{~N} / \mathrm{m}$. The steady-state droplet size distribution, calculated by the dispersion model developed, is employed for this analysis. Figure 4 illustrates the dropletwall interaction process. Figure $4 \mathrm{a}$ shows that how the velocities of droplets of different sizes change during the droplet compression stage.
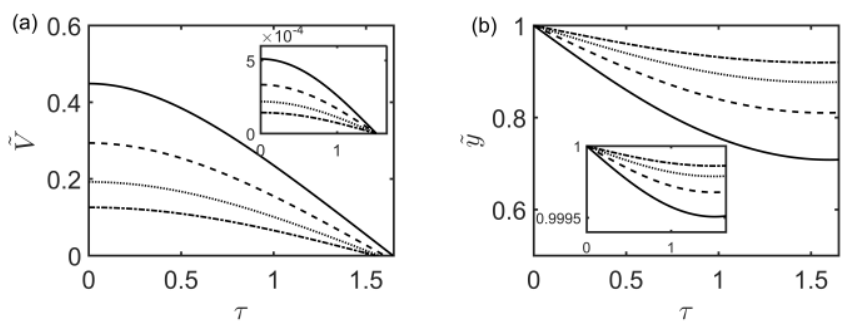

Figure 4. The dimensionless velocity (a) and the dimensionless distance from the droplet center to the wall surface (b) versus dimensionless time during the droplet compression stage. The droplet size decreases for the line, dashed line, dotted line and dash-dotted line, respectively. The results are shown for the four first larger droplets and the insets show the results of the four last small droplets.
Figure $4 \mathrm{~b}$ shows how the distance from the droplet center to the wall changes during the compression stage. The largest deformation is observed for the droplet of the largest size. However, even in this case, the maximum deformation value does not exceed $30 \%$ of the droplet radius. Thus, the assumption on the small droplet deformation accepted for modeling is satisfactory. Moreover, the fraction of larger droplets in the entire population is relatively small, whereas the deformation rapidly decreases with a reduction in droplet size.

For the same mixture, we also calculate the specific pipe surface, dynamically coated by droplets, for the different mean flow velocities $U=0.8,1,1.5$ and $2 \mathrm{~m} / \mathrm{s}$. Droplets are initially assumed to be monodispersed with the size $d_{o}=150 \mu \mathrm{m}$. Figure 5 illustrates the distribution of the sought parameters along a tubing of $4 \mathrm{~km}$ long. Only a negligible fraction of the pipe surface is dynamically coated with droplets. The droplet sizes decrease along a tubing due to their fragmentation. The droplet size reduction leads to an increase in the droplet number concentration causing an increase in the droplet-pipe wall collision frequency. As a result, the pipe surface area, coated with droplets, slowly increases along the tubing. In addition, the droplet coated surface expectedly increases with an increase in the mean flow velocity because of the two effects. First, droplet sizes decrease with an increase in the mean flow velocity due to a corresponding increase in the turbulence energy dissipation rate. Second, a higher mean flow velocity results in a larger droplet fluctuation velocity. An increase in the fluctuation velocity causes an enhancement in frequency of droplet-wall collisions as well as in stronger droplet deformations. These factors lead to an increase in a dropletwall contact area. A significant increase in the water holdup (see Figure 5b) leads to a relatively slight increase in the specific wall area dynamically coated by droplets. An increase in the holdup causes a reduction in the droplet breakup rate. Therefore, an effect of water holdup on the pipe area, dynamically coated by droplets, is relatively small.

The major outcome of our modeling is a demonstration that the effect of water dispersed in oil on the process of asphaltene deposition in a production tubing is negligible.
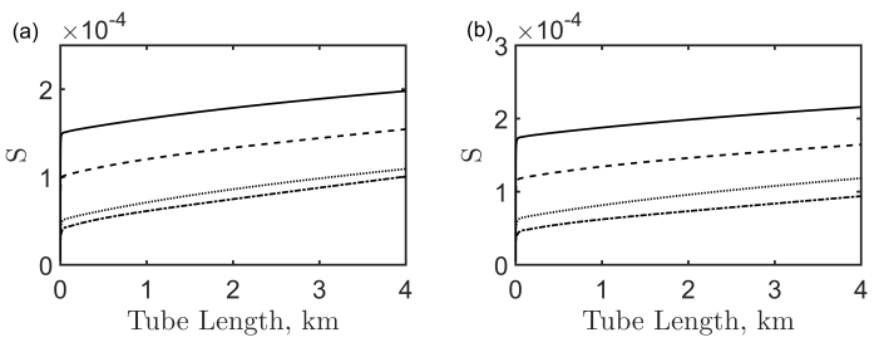

Figure 5. Distribution of the specific pipe wall area covered by droplets, along the pipe. Flow velocities: $U=0.8,1.0,1.5,2.0 \mathrm{~m} / \mathrm{s}$ corresponding to the dash-dotted, dotted line, dashed line and line, respectively. Water hold up: = $\phi=0.1$ (a) and 0.4 (b). 


\section{CONCLUSIONS}

An engineering model was developed to estimate the pipe wall surface area dynamically coated by droplets, which collide with the wall due to their fluctuations in a turbulent flow. An evolution of the droplet size distribution along production a tubing was modeled by a population balance equation. A droplet-wall collision process was modeled assuming that a droplet deformation in a single collision was small. It was also assumed that droplets follow the fluid in fluctuation motion caused by turbulence. The frequencies of the droplet-wall collisions were calculated assuming that the droplet fluctuation velocity distribution was Maxwellian. Calculations of the specific pipe surface area dynamically coated with water droplets, at different mean flow velocities and water holdups, showed that the coated specific area remains small and, therefore, the effect of produced water on asphaltene deposition can be ignored in practical computations of asphaltene deposition.

\section{ACKNOWLEDGMENT}

This research has been carried out at Université Laval, supported financially by the NSERC Engage grant. The authors acknowledge Schlumberger Ltd. for the support of this work.

\section{REFERENCES}

[1] D. Eskin, J. Ratulowski, K. Akbarzadeh, S. Pan, Can. J. "Modelling asphaltene deposition in turbulent pipeline flows", Chem. Eng. 2011.

[2] D. Eskin, J. Ratulowski, K. Akbarzadeh, S. Andersen, "Modeling of asphaltene deposition in a production tubing", AlChE J. 2012, 58.2936.

[3] J. Davies, "Drop sizes of emulsions related to turbulent energy dissipation rates", Chem. Eng. Sci. 1985, 40, 839.

[4] D. Eley, M. Hey, "Emulsions of water in asphaltene-containing oils 1. Droplet size distribution and emulsification rates", J. Symonds, Colloids Surf. 1988, 32, 87.

[5] A. S. Kurup, F. Vargas, J. Wang, J. Buckley, J. Creek, H. Subramani, W. Chapman, "Development and application of an asphaltene deposition tool (ADEPT) for well bores", Energy Fuels 2011, 25, 4506.

[6] G. Mansoori, "Modeling of asphaltene and other heavy organic depositions", J. Petrol. Sci. Eng 1997, 17, 101.

[7] Solsvik, J and Jakobsen, H, "On the solution of the population balance equation for bubbly flows using the high-order least squares method: implementation issues", Rev. Chem. Eng. 2013, 29, 63.

[8] Solsvik, $\mathbf{J}$ and Becker, $\mathbf{J}$ and Sheibat-Othman, $\mathrm{N}$ and Jakobsen, $\mathrm{H}$, "Population balance model: Breakage kernel parameter estimation to emulsification data”, Can. J. Chem. Eng., 2014, 6, 1082.

[9] C. Coulaloglou, L. Tavlarides, "Description of interaction processes in agitated liquid-liquid dispersions", Chem. Eng. Sci. 1977, 32, 1289.

[10] Prince, MJ and Blanch, HW, "Bubble coalescence and break-up in airsparged bubble columns", AlChE. J., 1190, 10, 1485.

[11] R. Kuboi, I. Komasawa, T. Otake, "Behavior of dispersed particles in turbulent liquid flow", J. Chem. Eng. Jpn. 1972, 5, 349. 